\title{
Numerical Integration of Nonlinear Wave Equations for General Relativity
}

\author{
Maurice H.P.M. van Putten * \\ CRSR \& Cornell Theory Center, Cornell University, Ithaca, NY 14853-6801, and \\ Department of Mathematics, MIT, Cambridge, MA 02139.
}

(October 5, 2018)

\begin{abstract}
A second-order numerical implementation is given for recently derived nonlinear wave equations for general relativity. The Gowdy $\mathrm{T}^{3}$ cosmology is used as a test bed for studying the accuracy and convergence of simulations of one-dimensional nonlinear waves. The complete freedom in space-time slicing in the present formalism is exploited to compute in the Gowdy line-element. Second-order convergence is found by direct comparison of the results with either analytical solutions for polarized waves, or solutions obtained from Gowdy's reduced wave equations for the more general unpolarized waves. Some directions for extensions are discussed.
\end{abstract}

PACS numbers: 04.25.Dm, 04.30.Nk

*Electronic address: mvp@schauder.mit.edu 


\section{INTRODUCTION}

The gravitational wave observatories LIGO and VIRGO [11,1] presently under construction have given added impetus towards accurate prediction of gravitational wave forms from binary coalescence of neutron stars and black holes. The late stages of the spiral infall are predominantly targeted through large scale simulations in numerical relativity. The gravitational wave interactions have motivated a description of gravity by nonlinear wave equations in a tetrad approach ( [9]). In this formulation, the strictly hyperbolic nature of the equations is independent of the particular choice of foliation. The foliation is governed by four tetrad lapse functions which are algebraically related to the more familiar Hamiltonian lapse and shift functions.

In this paper, a numerical implementation of the nonlinear wave equations is given, and the performance is studied in the Gowdy $\mathrm{T}^{3}$ cosmology. The Gowdy $\mathrm{T}^{3}$ is a good test bed for one-dimensional nonlinear wave motion of both polarized and unpolarized waves. The numerical scheme is one-dimensional, second-order in time and spectrally accurate in space. The $\mathrm{SO}(3,1, \mathbf{R})$-connections are evolved by implementation of the four-divergence of the Riemann tensor and the Lorentz gauge on the connections. The tetrad elements are evolved by the equations of structure, and treated as a system of ordinary differential equations in which the fundamental matrix is a finite Lorentz transformation. The Gowdy $\mathrm{T}^{3}$ line-element has been incorporated in the numerical scheme, so as to enable direct comparison of the computed solution with either analytical or numerical solutions obtained from Gowdy's reduced wave equations. Convergence results are presented for both polarized and unpolarized Gowdy waves.

The nonlinear wave equations for the connections, $\omega_{a \mu \nu}$, of the tetrad elements, $\left\{\left(e_{\mu}\right)_{a}\right\}$ satisfy nonlinear wave equations of the Yang-Mills type. They follow from a Lorentz gauge on the connections. In vacuo, they simplify to

$$
\square \omega_{a \mu \nu}-\left[\omega^{c}, \nabla_{a} \omega_{c}\right]_{\mu \nu}=0
$$

Here, $\square=\hat{\nabla}^{c} \hat{\nabla}_{c}$ with $\hat{\nabla}_{a}$ the $\operatorname{SO}(3,1, \mathbf{R})$-gauge covariant derivative satisfying $\hat{\nabla}_{a}\left(e_{\mu}\right)_{b}=$ 
$\nabla_{a}\left(e_{\mu}\right)_{b}+\omega_{a \mu}^{\gamma}\left(e_{\gamma}\right)_{b}=0$. The tetrad elements define the metric by $g_{a b}=\left(e_{\mu}\right)_{a}\left(e^{\mu}\right)_{b}$. Here, contraction over the Greek indices is through $\eta_{\mu \nu}=\operatorname{diag}(-1,1,1,1)$. Initial data in this second-order formalism includes the initial data for the familiar first-order Hamiltonian formalism. Additional initial data are for example initial values for the tetrad; with sufficient smoothness these data generate initial data for the connections and the Riemann tensor. A procedure for obtaining smooth initial tetrad data from the metric is included.

In Section 2, the Gowdy waves and their reduced wave equations are discussed. Section 3 presents the integration scheme, together with the procedure for computing initial data for the tetrad elements. Section 4 discusses the gauge conditions in the Gowdy line-element. Discussion of the simulations and conclusions are given in Section 5.

\section{GOWDY WAVES}

Gowdy cosmologies describe an extensively studied class of universes with compact spacelike hypersurfaces with two Killing vectors, $\partial_{\sigma}$ and $\partial_{\delta}$. By choice of boundary conditions, the space-like hypersurfaces are homeomorphic to either the three-torus, the three-handle or the three-sphere. The three-torus describes a semi-infinite time-evolution of universes collapsing into, or beginning with a singularity.

The Gowdy three-torus cosmology has recently been investigated numerically by Berger and Moncrief [3], and can be given in the line-element

$$
d s^{2}=e^{-\frac{\lambda}{2}} e^{\frac{\tau}{2}}\left(-e^{-2 \tau} \mathrm{d} \tau^{2}+\mathrm{d} \theta^{2}\right)+\mathrm{d} \Sigma^{2},
$$

where

$$
\mathrm{d} \Sigma^{2}=e^{-\tau}\left[e^{P} \mathrm{~d} \sigma^{2}+2 e^{P} Q \mathrm{~d} \sigma \mathrm{d} \delta+\left(e^{P} Q^{2}+e^{-P}\right) \mathrm{d} \delta^{2}\right] .
$$

Here $\lambda=\lambda(\tau, \theta), P=P(\tau, \theta)$ and $Q=Q(\tau, \theta)$, by invariance in the angular coordinates $\sigma$ and $\delta$.

In small amplitude waves, $e^{-\tau} P$ and $e^{-\tau} Q$ are the amplitudes of the $\epsilon_{+}$and $\epsilon_{\times}$gravitational wave polarization tensors (Eqn. 3.17 in [3]). The quantities $P$ and $Q$ satisfy Gowdy's 
reduced wave equations, which may be given as [3]:

$$
\begin{aligned}
& Q_{\tau \tau}=e^{-2 \tau} Q_{\theta \theta}-2\left(P_{\tau} Q_{\tau}-e^{-2 \tau} P_{\theta} Q_{\theta}\right), \\
& P_{\tau \tau}=e^{-2 \tau} P_{\theta \theta}+e^{-2 P}\left(Q_{\tau}^{2}-e^{-2 \tau} Q_{\theta}^{2}\right) .
\end{aligned}
$$

The $\theta$-momentum and Hamiltonian constraints, respectively, are given by

$$
\begin{aligned}
& \lambda_{\theta}=2\left(P_{\theta} P_{\tau}+e^{2 P} Q_{\theta} Q_{\tau}\right), \\
& \lambda_{\tau}=\left[P_{\tau}^{2}+e^{-2 \tau} P_{\theta}^{2}+e^{2 P}\left(Q_{\tau}^{2}+e^{-2 \tau} Q_{\theta}^{2}\right)\right] .
\end{aligned}
$$

Notice that the wave equations (4) evolve in an unconstraint manner with respect to (5).

These equations serve two purposes. They are used to generate initial data, and can be readily integrated for obtaining reference solutions against which the simulations can be compared. A simplectic integration scheme has been given by [3]. For reasons of convenience, a leap frog integration scheme in combination with spectrally accurate spatial differentiation by the Fast Fourier Transform has been used here. Thus, general Gowdy waves are at hand with high accuracy as a reference in studying the simulations in the tetrad approach. In the polarized case, furthermore, analytical solutions are available.

\section{A. A polarized Gowdy wave}

Polarized Gowdy wave result in the special case $Q=0$, so that the Gowdy line-element becomes

$$
d s^{2}=e^{\frac{\tau-\lambda}{2}}\left(-e^{-2 \tau} \mathrm{d} \tau^{2}+\mathrm{d} \theta^{2}\right)+e^{-\tau}\left[e^{P} \mathrm{~d} \sigma^{2}+e^{-P} \mathrm{~d} \delta^{2}\right]
$$

The reduced wave equation (田) is linear in $P$, and can be solved by the method of separation of variables, giving [3]

$$
P(\tau, \theta)=\Sigma_{n} Z_{0}\left(n e^{-\tau}\right)\left(a_{n} \cos n \theta+b_{n} \sin n \theta\right),
$$

where $Z_{0}$ is a solution to Bessel's equation of order zero, and where the linearly growing solution $P=P_{0}+\tau P_{1}\left(P_{0}\right.$ and $P_{1}$ constant $)$ has been suppressed. 
A polarized Gowdy which evolves towards a singularity is exemplified by (Equation (4.2) in [3])

$$
P_{0}(\tau, \theta)=Y_{0}\left(e^{-\tau}\right) \cos \theta
$$

where $Y_{0}$ is the Bessel function of the second kind of order zero. With $Q=0$ the Hamiltonian constraints for $\lambda$ at $\tau=0$ become [3]

$$
\begin{aligned}
& \lambda_{\theta}=2 P_{\theta} P_{\tau}, \\
& \lambda_{\tau}=P_{\tau}^{2}+e^{-2 \tau} P_{\theta}^{2},
\end{aligned}
$$

so that (8) implies

$$
\begin{aligned}
& \lambda(\tau, \theta)=\frac{1}{2} Y_{0}\left(e^{-\tau}\right) Y_{1}\left(e^{-\tau}\right) e^{-\tau} \cos 2 \theta+T(\tau), \\
& T(\tau)=\frac{1}{2} \int_{e^{-\tau}}^{1}\left\{Y_{0}^{\prime 2}(t)+Y_{0}^{2}(t)\right\} t \mathrm{~d} t .
\end{aligned}
$$

Equations (8) and (10) provide for initial data for simulations, an are an analytical reference solution for polarized waves. The computational procedure to treat the integral $T(\tau)$ is outlined in the Appendix.

\section{B. Unpolarized Gowdy waves}

Unpolarized Gowdy waves follow from general initial conditions on the $P(\tau, \theta)$ and $Q(\tau, \theta)$. Following the earlier computations of Berger et el. [3], we consider

$$
\begin{array}{ll}
P(0, \theta)=0, & \partial_{\tau} P(\tau, \theta)=A \cos (\theta), \\
Q(0, \theta)=B \cos (\theta), & \partial_{\tau} Q(\tau, \theta)=0
\end{array}
$$

where $A$ and $B$ are constants. We then have the expansions

$$
\begin{aligned}
& P(\tau, \theta) \sim A \tau \cos \theta-\frac{1}{2} \tau^{2} B^{2} \sin ^{2} \theta \\
& Q(\tau, \theta) \sim\left(1-\frac{1}{2} B \tau^{2}\right) \cos \theta \\
& \lambda(\tau, \theta) \sim \tau\left(A^{2} \cos ^{2} \theta+B^{2} \sin ^{2} \theta\right)-\tau^{2} B^{2} \sin ^{2} \theta
\end{aligned}
$$

valid to second order in $\tau$. These expansions are used to compute initial data for the simulations. 


\section{INTEGRATION SCHEME}

The integration scheme combines an evolution scheme for the tetrad connections $\omega_{a \mu \nu}$ and an evolution scheme for the tetrad legs $\left(e_{\mu}\right)^{b}$. The scheme is one-dimensional, second-order accurate in the time-coordinate $\tau$ and spectrally accurate in the space-coordinate $\theta$. The scheme uses a straightforward leap frog time-stepping algorithm, and, in view of periodicity in $\theta$, differentiation by the Fast Fourier Transform. In this fashion, the errors are essentially due to the time-stepping algorithm.

In what follows, we shall work with both tensors and their densities, denoted by a tilde, e.g., $\tilde{\phi}=\sqrt{-g} \phi$. Time is discretized as $\tau_{n}=n \Delta \tau$, indicated by a superscript: $\phi\left(\tau_{n}\right)=\phi^{n}$. Indices $a, b$ will refer to all four space-time indices, and $p, q, r, s, u, v$ to the spatial $\theta, \delta$ and $\sigma$ only.

\section{A. Evolution of the connections $\omega_{a \mu \nu}$}

The wave equations for $\omega_{a \mu \nu}$ are implemented directly through the divergence of the Riemann tensor, its representation in terms of the connections and the Lorentz gauge condition $c_{\mu \nu}=\nabla^{a} \omega_{a \mu \nu}=0$.

The integration is based on the four-divergence of the Riemann tensor in the tetrad ap-

proach, $\hat{\nabla}^{a} R_{a b \mu \nu}=0$. In coordinate notation, this gives $\partial_{a} \tilde{R}_{\mu \nu}^{a b}+\left[\omega_{a}, \tilde{R}^{a b}\right]_{\mu \nu}=0$. Application of leap frog time-stepping gives the iterations

$$
\begin{aligned}
\left(\tilde{R}_{\mu \nu}^{\tau r}\right)^{n+1}= & \left(\tilde{R}_{\mu \nu}^{\tau r}\right)^{n-1} \\
& -2 \Delta \tau\left(\partial_{p} \tilde{R}_{\mu \nu}^{p r}+\left[\omega_{a}, \tilde{R}^{a r}\right]_{\mu \nu}\right)^{n}
\end{aligned}
$$

Evolution equations for the connections $\omega_{p \mu \nu}$ (where $p$ is a spatial coordinate) then follow from the identity $R_{\tau p \mu \nu}=\partial_{\tau} \omega_{p \mu \nu}-\partial_{p} \omega_{\tau \mu \nu}+\left[\omega_{\tau}, \omega_{p}\right]_{\mu \nu}$. Application of leap frog time-stepping gives

$$
\begin{aligned}
\left(\omega_{p \mu \nu}\right)^{n+1}= & \left(\omega_{p \mu \nu}\right)^{n-1} \\
& +2 \Delta \tau\left(R_{\tau p \mu \nu}+\partial_{p} \omega_{\tau \mu \nu}-\left[\omega_{\tau}, \omega_{p}\right]_{\mu \nu}\right)^{n}
\end{aligned}
$$


Evolution of the remaining $\omega_{\tau \mu \nu}$ are determined from the Lorentz gauge conditions $c_{\mu \nu}=$ $\nabla_{c} \omega_{\mu \nu}^{c}=0$, and hence

$$
\begin{aligned}
\left(g^{\tau \tau} \tilde{\omega}_{\tau \mu \nu}\right)^{n+1} & +\left(g^{\tau p} \tilde{\omega}_{p \mu \nu}\right)^{n+1}=\left(\tilde{\omega}_{\mu \nu}^{\tau}\right)^{n-1} \\
& -2 \Delta \tau \partial_{p}\left(\tilde{\omega}_{\mu \nu}^{p}\right)^{n} .
\end{aligned}
$$

Note that $R_{\tau p \mu \nu}^{n}$ appears in Step (b) of the iteration scheme. An update $R_{\tau p \mu \nu}^{n+1}$ is algebraically related to $\left(R_{\mu \nu}^{\tau p}\right)^{n+1}$ and $\left(R_{p q \mu \nu}\right)^{n+1}$, the first of which follows from Step (a) and the second of which follows from Step (b). Suppressing momentarily the tetrad idices $\mu \nu$ and the time-label $n+1$, we have

$$
\begin{aligned}
R_{\tau p} & =g_{\tau c} g_{d p} R^{c d}=2 g_{\tau[\tau} g_{r] p} R^{\tau r}+g_{\tau[s} g_{r] p} R^{s r} \\
& =2 g_{\tau[\tau} g_{r] p} R^{\tau r}+g_{\tau[s} g_{r] p} g^{s a} g^{b r} R_{a b} \\
& =A_{p}^{v} R_{\tau v}+B_{p},
\end{aligned}
$$

were

$$
\begin{aligned}
& A_{p}^{v}=2 g_{\tau[s} g_{r] p} g^{\tau s} g^{r v}, \\
& B_{p}=2 g_{\tau[\tau} g_{r] p} R^{\tau r}+g_{\tau[s} g_{r] p} g^{u s} g^{r v} R_{u v}
\end{aligned}
$$

Note that $A_{p}^{v}$ and the second term in $B_{p}$ is due to a shift, $g_{\tau p}$; in particular, $A_{p}^{v}=0$ whenever $g_{\tau p}=0$. It follows that

$$
\left(\delta_{p}^{v}-A_{p}^{v}\right) R_{\tau v}=B_{p} .
$$

As a system of $3 \times 3$ equations, this is readily inverted. With all quantities at $t_{n+1}=(n+1) \Delta \tau$, (18) determines updates $\left(R_{\tau p \mu \nu}\right)^{n+1}$ from the results of the previous steps.

\section{B. Evolution of the tetrad legs $\left(e_{\mu}\right)^{b}$}

The tetrad legs satisfy the equations of structure

$$
\partial_{[a}(e \mu)_{b]}=\left(e_{\nu}\right)_{[b} \omega_{a] \mu}{ }^{\nu} .
$$

Clearly, these equations leave the tetrad lapse functions $N_{\mu}=\left(e_{\mu}\right)_{\tau}$ as free variables. They are related to the Hamiltonian lapse and shift functions $\left(N, N_{p}\right)$ through 


$$
g_{a \tau}=N_{\alpha}\left(e^{\alpha}\right)_{a}=\left(N_{p} N^{p}-N^{2}, N_{p}\right) .
$$

These tetrad lapse functions govern the evolution of the tetrad legs in the equations of structure:

$$
\partial_{\tau}\left(e_{\mu}\right)_{b}+\omega_{\tau \mu}{ }^{\nu}\left(e_{\nu}\right)_{b}=\partial_{b} N_{\mu}+\omega_{b \mu}{ }^{\nu} N_{\nu} \equiv \hat{\partial}_{b} N_{\mu} .
$$

Thus, conditions on $g_{a \tau}$ from the line-element (2) result in a system of implicit equations for the lapse functions $N_{\mu}$, as discussed in Section IV.

Integration of (21) can be written using the fundamental matrix $\Lambda_{\mu}^{\nu}\left(\tau ; \tau_{0}\right)$ : a finite Lorentz transformation satisfying

$$
\left\{\begin{array}{l}
\Lambda_{\mu}^{\nu}(\tau, \tau)=\delta_{\mu}^{\nu}, \\
\partial_{\tau} \Lambda_{\mu}^{\nu}\left(\tau ; \tau_{0}\right)=-\omega_{\tau \mu}^{\alpha}(\tau) \Lambda_{\alpha}^{\nu}\left(\tau ; \tau_{0}\right),
\end{array}\right.
$$

with which the solution to (21) is

$$
\begin{aligned}
\left(e_{\mu}\right)_{b}(\tau)= & \Lambda_{\mu}^{\nu}\left(\tau ; \tau_{0}\right)\left(e_{\nu}\right)_{b}\left(\tau_{0}\right) \\
& +\int_{\tau_{0}}^{\tau} \Lambda_{\mu}^{\nu}(\tau ; s) \hat{\partial}_{b} N_{\nu}(s) \mathrm{d} s .
\end{aligned}
$$

Here, only the $\tau$-dependence has been made explicit. Indeed, if the convolution integral on the right hand-side were to vanish, the evolution of the tetrad becomes a pure $\mathrm{SO}(3,1, \mathbf{R})$ gauge transformation, leaving the metric $g_{a b}(\tau)=\left(e_{\alpha}\right)_{a}(\tau)\left(e^{\alpha}\right)_{b}(\tau)$ constant. The general representation (23) shows explicitly that the evolution of the metric takes place in the (flat) tangent bundle of the four-dimensional, physical manifold. The integral in (23) is implemented using Gaussian integration, including a Taylor expansion for the integrand as provided by the updates in Section 3.1. Thus, in the case of given connections, the tetrad evolution is carried out with machine precision. In general, a Taylor expansion of $\omega_{a \mu \nu}$ up to its $n$-th derivatives at $t_{n}$ in (23) provides an update of the tetrad legs with $n+1$-th order accuracy. 


\section{Equations for $\Lambda_{\mu}^{\nu}\left(\tau ; \tau_{0}\right)$}

In the Gowdy line-element, $\Gamma_{\tau c}^{d}, \Gamma_{\theta c}^{d}$ and $R_{\tau \theta c}^{d}$ all have a $2 \times 2$ block diagonal structure as matrices in $c, d$. Initial data for the tetrad (Section D) can be chosen with $\left(e_{\mu}\right)_{b}$ likewise, when regarded as a matrix in $\mu, b$. This obtains $\omega_{\tau \mu \nu}, \omega_{\theta \mu \nu},\left[\omega_{\tau}, \omega_{\theta}\right]_{\mu \nu}$ and $R_{\tau \theta \mu \nu}$ in block diagonal form at $\tau=0$. Consider now the evolution problem for the connections in terms of $\partial_{\tau} \omega_{\theta \mu \nu}=\partial_{\theta} \omega_{\tau \mu \nu}+R_{\tau \theta \mu \nu}-\left[\omega_{\tau}, \omega_{\theta}\right]$ and the Lorentz gauge, $c_{\mu \nu}=0$. Here, $R_{\tau \theta \mu \nu}=$ $R_{\tau \theta c d}\left(e_{\mu}\right)^{c}\left(e_{\nu}\right)^{d}$, where (the structure of) $R_{\tau \theta c d}$ is as mentioned above, and where the tetrad elements satisfy (21) with $N_{\mu}=(*, *, 0,0)$ (Section IV). Consistent evolution is obtained in which the block diagonal structure of the forementioned tensor elements is preserved with

$$
\omega_{\tau \mu}^{\nu}=\left(\begin{array}{cc}
a \sigma & 0 \\
0 & b \epsilon
\end{array}\right),
$$

where $a=a(\tau, \theta)$ and $b=b(\tau, \theta)$, and

$$
\sigma=\left(\begin{array}{ll}
0 & 1 \\
1 & 0
\end{array}\right), \epsilon=\left(\begin{array}{ll}
0 & -1 \\
1 & 0
\end{array}\right) .
$$

By explicit exponentiation, the Lorentz transformation of boosts on the $\left(e_{T}\right)^{b}$ and $\left(e_{\Theta}\right)^{b}$, and rotations on $\left(e_{\Sigma}\right)^{b}$ and $\left(e_{\Delta}\right)^{b}$ is therefore

$$
\Lambda_{\mu}^{\nu}=\left(\begin{array}{cc}
K & 0 \\
0 & L
\end{array}\right) .
$$

Here, we adapt the nomenclature of [2], with the 2x2 matrices $K$ and $L$ given by

$$
K_{\mu}^{\nu}=\left(\begin{array}{cc}
\cosh \phi & \sinh \phi \\
\sinh \phi & \cosh \phi
\end{array}\right)(\mu, \nu=T, \Theta),
$$

with

$$
\phi\left(\tau ; \tau_{0}\right)=\int_{\tau}^{\tau_{0}} a(s) \mathrm{d} s, \psi\left(\tau ; \tau_{0}\right)=\int_{\tau}^{\tau_{0}} b(s) \mathrm{d} s
$$




\section{Initial data and choice of tetrad}

The integration scheme requires $\omega_{a \mu \nu}$ and $\partial_{\tau} \omega_{a \mu \nu}$ for obtaining initial values of the Riemann tensor on $\tau=0$. For the polarized Gowdy wave $(Q=0)$, we can simply use

$$
\begin{aligned}
& \left(e_{T}\right)^{b}=\left(e^{\frac{\lambda}{4}}, 0,0,0\right), \\
& \left(e_{\Theta}\right)^{b}=\left(0, e^{\frac{\lambda}{4}}, 0,0\right), \\
& \left(e_{\Sigma}\right)^{b}=\left(0,0, e^{-\frac{P_{0}}{2}}, 0\right), \\
& \left(e_{\Delta}\right)^{b}=\left(0,0,0, e^{\frac{P_{0}}{2}}\right) .
\end{aligned}
$$

In case of the full Gowdy metric $(P, Q \neq 0)$, care must be given to the choice of initial tetrad to ensure sufficient differentiability for obtaining smooth connections $\omega_{a \mu \nu}$ and Riemann tensor $R_{a b \mu \nu}$. The tetrad legs for the $\tau-\theta$ coordinate plane can be chosen as before,

$$
\begin{aligned}
& \left(e_{T}\right)^{b}=\left(e^{\frac{\lambda}{4}}, 0,0,0\right), \\
& \left(e_{\Theta}\right)^{b}=\left(0, e^{\frac{\lambda}{4}}, 0,0\right) .
\end{aligned}
$$

The tetrad legs for the $\sigma-\delta$ coordinate plane,

$$
E=\left(\begin{array}{cc}
\left(e_{\Sigma}\right)_{\sigma} & \left(e_{\Delta}\right)_{\sigma} \\
\left(e_{\Sigma}\right)_{\delta} & \left(e_{\Delta}\right)_{\delta}
\end{array}\right)
$$

must satisfy $E E^{T}=G, E G^{-1} E^{T}=I$, where $I$ is the $2 \times 2$ identity matrix and $G$ is the matrix with the metric components

$$
G=\left(\begin{array}{ll}
g_{\sigma \sigma} & g_{\sigma \delta} \\
g_{\sigma \delta} & g_{\delta \delta}
\end{array}\right)=e^{-\tau+P}\left(\begin{array}{ll}
1 & Q \\
Q & Q^{2}+e^{-2 P}
\end{array}\right) .
$$

$E$ can be defined uniquely as the symmetric positive definite square root (cf. [4] ) of $G$. To this end, let

$$
A(\phi)=\left(\begin{array}{rr}
\cos \phi & -\sin \phi \\
\sin \phi & \cos \phi
\end{array}\right)
$$


denote both the matrix containing the tangents to the tetrad legs, and the rotation matrix on the tetrad indices $\mu=\sigma, \delta$. Then

$$
E=A(\phi)\left(\begin{array}{cc}
\lambda_{+}^{\frac{1}{2}} & 0 \\
0 & \lambda_{-}^{\frac{1}{2}}
\end{array}\right) A(-\phi),
$$

where the eigenvalues $\lambda_{ \pm}$of $G$ can be given in terms of $z=\frac{1}{2}\left(Q^{2}+e^{-2 P}-1\right)$ and $r=\sqrt{Q^{2}+z^{2}}$ as

$$
\lambda_{ \pm}=(1+z \pm r) e^{-\tau+P}
$$

The rotation angle $\phi$ follows from the equations

$$
z \sin 2 \phi+Q \cos 2 \phi=0, z+r \cos 2 \phi=0 .
$$

The second equation in (37) gives $\cos 2 \phi=-\frac{z}{r}$, and hence $\sin 2 \phi=\frac{Q}{r}$. Without loss of generality, $\cos \phi \geq 0$, so that $0 \leq \phi \leq \frac{\pi}{2}$ if $Q \geq 0$, and $-\frac{\pi}{2} \leq \phi<0$ if $Q<0$. The additional rotation $A(-\phi)$ on the tetrad indices in the right hand-side of (35) effectively regularizes $E$ as $G$ approaches the 2x2 identity matrix (when $P$ and $Q$ become small, in which case $\phi$ becomes ill-defined).

The connections $\omega_{a \mu \nu}$ are now computed using its metric definition

$$
\omega_{a \mu \nu}=\left(e_{\mu}\right)_{c} \partial_{a}\left(e_{\nu}\right)^{c}+\Gamma_{a b}^{c}\left(e_{\mu}\right)_{c}\left(e_{\nu}\right)^{b}
$$

Initial data for the Riemann tensor of Section IIIa now follow.

\section{GAUGE CONDITIONS FOR GOWDY WAVE}

The Gowdy line-element prescribes a certain slicing of space-time, which are incorporated in the simulations to enable comparison with the analytical solution for the polarized and pseudo-unpolarized Gowdy waves, and the reference solutions obtained by integration of the reduced wave equations (雨) and Hamiltonian constraints (5).

The Gowdy line-element (2) has algebraic slicing conditions 


$$
\left\{\begin{array}{l}
g_{\tau \tau}=-e^{-2 \tau} g_{\theta \theta} \\
g_{\tau \theta}=0 \\
g_{\tau \sigma}=0 \\
g_{\tau \delta}=0
\end{array}\right.
$$

We have

$$
\begin{aligned}
& g_{\tau \tau}=\left(e_{\alpha}\right)_{\tau}\left(e^{\alpha}\right)_{\tau}=-N_{T}^{2}+N_{\Theta}^{2}, \\
& g_{\theta \theta}=\left(e_{\alpha}\right)_{\theta}\left(e^{\alpha}\right)_{\theta}=-\left(e_{T}\right)_{\theta}^{2}+\left(e_{\Theta}\right)_{\theta}^{2}, \\
& g_{\tau \theta}=\left(e_{\alpha}\right)_{\tau}\left(e^{\alpha}\right)_{\theta}=-N_{T}\left(e_{T}\right)_{\theta}+N_{\Theta}\left(e_{\Theta}\right)_{\theta} .
\end{aligned}
$$

The shift condition $g_{\tau \theta}=0$ gives

$$
\frac{N_{\Theta}}{N_{T}}=\frac{\left(e_{T}\right)_{\theta}}{\left(e_{\Theta}\right)_{\theta}}
$$

so that

$$
g_{\theta \theta}=\left(e_{\Theta}\right)_{\theta}^{2}\left(1-\frac{\left(e_{T}\right)_{\theta}^{2}}{\left(e_{\Theta}\right)_{\theta}^{2}}\right)=\left(e_{\Theta}\right)_{\theta}^{2}\left(1-\frac{N_{\Theta}^{2}}{N_{T}^{2}}\right) \text {. }
$$

Because also

$$
g_{\tau \tau}=-N_{T}^{2}\left(1-\frac{N_{\Theta}^{2}}{N_{T}^{2}}\right),
$$

the lapse condition $g_{\tau \tau}=-e^{-2 \tau} g_{\theta \theta}$ becomes

$$
N_{T}^{2}=e^{-2 \tau}\left(e_{\Theta}\right)_{\theta}^{2}
$$

We thus have the following conditions on the tetrad lapse functions

$$
\left\{\begin{array}{l}
N_{T}=-e^{-\tau}\left(e_{\Theta}\right)_{\theta}=-e^{-\tau} \sigma_{T}^{\gamma}\left(e_{\gamma}\right)_{\theta} \\
N_{\Theta}=-e^{-\tau}\left(e_{T}\right)_{\theta}=-e^{-\tau} \sigma_{\Theta}^{\gamma}\left(e_{\gamma}\right)_{\theta} \\
N_{\Sigma}=0 \\
N_{\Delta}=0
\end{array}\right.
$$

Upon substitution of (45) into the equations of structure (23), two implicit equations for the $\left(e_{\Theta}\right)_{\theta}$ and $\left(e_{T}\right)_{\theta}$ result. 


\section{A. Solution of gauge conditions}

The nontrivial $\tau-\theta$ gauge conditions in (45) are separated from the trivial gauge conditions on the $\sigma-\delta$ coordinates. Our starting point, therefore, is

$$
\left(e_{\mu}\right)_{\theta}(\tau, \theta)=K_{\mu}^{\nu}\left(\tau ; \tau_{0}, \theta\right) \eta_{\nu}(\tau, \theta),(\mu, \nu=T, \Theta)
$$

and to work in the $\tau-\theta$ sector only. In what follows, Greek tetrad indices run through $T, \Theta$. Also, the spatial $(\theta-)$ dependence will made explicit only when needed.

The original gauge conditions (39) are given in terms of the metric, which are invariants under pointwise $K$ transformations applied simultaneously to both $\left(e_{T}\right)^{b}$ and $\left(e_{\Theta}\right)^{b}$. Upon substitution of (46) in (45), therefore, $K$ can be factored out, leaving a linear equation for $\eta_{\mu}$.

Expressed in (46), the gauge conditions (45) become

$$
N_{\mu}=-e^{-\tau} \sigma_{\mu}^{\nu} K_{\nu}^{\gamma} \eta_{\gamma}
$$

Substitution of (46) and (47) into the equations of structure (21) gives two implicit equations for $\eta_{\mu}=\left(\eta_{T}, \eta_{\Theta}\right)$. Note that

$$
\begin{aligned}
& \omega_{\theta \mu}^{\nu}(\tau, \theta) \quad=\beta(\tau, \theta) \sigma_{\mu}^{\nu} \\
& \partial_{\theta} K_{\mu}^{\nu}\left(\tau ; \tau_{0}, \theta\right)=\sigma_{\mu}^{\gamma} K_{\gamma}^{\nu}\left(\tau ; \tau_{0}, \theta\right) \alpha\left(\tau ; \tau_{0}, \theta\right),
\end{aligned}
$$

where $\beta=\beta(\tau, \theta)$ is a coefficient function, and $\alpha\left(\tau ; \tau_{0}, p\right)=\int_{\tau}^{\tau_{0}} \partial_{\theta} a(s, p) \mathrm{d} s$. Together with $\partial_{t} K_{\mu}^{\nu}=-a \sigma_{\mu}^{\alpha} K_{\alpha}^{\nu}$ from the second equation in (22), and the algebraic properties $\sigma_{\mu}^{\gamma} \Lambda_{\gamma}^{\nu}=$ $\Lambda_{\mu}^{\gamma} \sigma_{\gamma}^{\nu}$ and $\sigma_{\mu}^{\gamma} \sigma_{\gamma}^{\nu}=\delta_{\mu}^{\nu}$, the linear equation for $\eta_{\mu}$ follows:

$$
\partial_{\tau} \eta_{\mu}+e^{-\tau}\left(\sigma_{\mu}^{\nu} \partial_{\theta} \eta_{\nu}+c\left(\tau, \tau_{0}\right) \eta_{\mu}\right)=0
$$

where $c\left(\tau, \tau_{0}\right):=\beta(\tau)+\alpha\left(\tau ; \tau_{0}\right)$. Because $\alpha(\tau ; \tau) \equiv 0, c\left(\tau, \tau_{0}\right)$ combines $\omega_{\theta \mu}^{\nu}$ with $\partial_{\theta} \omega_{\tau \mu}^{\nu}$ to first and second order in $\left(\tau-\tau_{0}\right)$, respectively.

Momentarily suppressing the tetrad index $\mu$, we now look for a solution to (49) in

$$
\eta=\eta_{0}+\left(\tau-\tau_{0}\right) \eta_{1}+\frac{1}{2}\left(\tau-\tau_{0}\right)^{2} \eta_{2}+\frac{1}{3}\left(\tau-\tau_{0}\right)^{3} \eta_{3}+\cdots
$$


where each $\eta_{k}=\eta_{k}(\theta)$ has only $\theta$-dependence. Similarly, we write

$$
\begin{gathered}
e^{\tau}=e^{\tau_{0}}+\left(\tau-\tau_{0}\right) e^{\tau_{0}}+\frac{1}{2 !}\left(\tau-\tau_{0}\right)^{2} e^{\tau_{0}}+\cdots, \\
c\left(\tau, \tau_{0}\right)=c_{0}+\left(\tau-\tau_{0}\right) c_{1}\left(\tau_{0}\right)+\left(\tau-\tau_{0}\right)^{2} c_{2}\left(\tau_{0}\right)+\cdots .
\end{gathered}
$$

Expansions (50) and (51) can substituted into (49); matching coefficients gives

$$
\sigma_{\mu}^{\nu} \partial_{\theta} \eta_{\nu 0}+c_{0} \eta_{0}=0
$$

for $k=0$, and

$$
e^{\tau_{0}} \sum_{l=0}^{k} \frac{1}{l !} \eta_{k+1-l}+\frac{1}{k} \sigma_{\mu}^{\nu} \partial_{\theta} \eta_{\nu k}+c_{k} \eta_{0}+\sum_{l=0}^{k-1} \frac{1}{k-l} c_{l} \eta_{k-l}=0
$$

for $k \geq 1$, i.e.,

$$
\begin{aligned}
\eta_{k+1}+ & \sum_{l=1}^{k} \frac{1}{l !} \eta_{k+1-l}+e^{-\tau_{0}}\left(\frac{1}{k} \sigma_{\mu}^{\nu} \partial_{\theta} \eta_{\nu k}\right. \\
& \left.+c_{k} \eta_{0}+\sum_{l=0}^{k-1} \frac{1}{k-l} c_{l} \eta_{k-l}\right)=0
\end{aligned}
$$

The first few terms are

$$
\left\{\begin{aligned}
\eta_{\mu 0}= & \left(e_{\mu}\right)_{\theta}\left(\tau_{0}\right) \\
\eta_{\mu 1}= & -e^{-\tau_{0}}\left(\sigma_{\mu}^{\nu} \partial_{\theta} \eta_{\nu 0}+c_{0} \eta_{\mu 0}\right) \\
\eta_{\mu 2}= & -\eta_{\mu 1}-e^{-\tau_{0}}\left(\sigma_{\mu}^{\nu} \partial_{\theta} \eta_{\nu 1}+c_{1} \eta_{\mu 0}+c_{0} \eta_{\mu 1}\right) \\
\eta_{\mu 3}= & -\eta_{\mu 2}-\frac{1}{2 !} \eta_{\mu 1} \\
& -e^{-\tau_{0}}\left(\frac{1}{2} \sigma_{\mu}^{\nu} \partial_{\theta} \eta_{\nu 2}+c_{2} \eta_{\mu 0}+c_{1} \eta_{\mu 1}+\frac{1}{2} c_{0} \eta_{\mu 2}\right) \\
\cdots= & \cdots
\end{aligned}\right.
$$

This series expansion has been implemented numerically using a numerical cut-off of 1.D-12, thereby maintaining the Gowdy line-element within machine accuracy.

\section{RESULTS AND CONCLUSIONS}

The performance of the numerical implementation has been studied by varying both space and time discretization. Verification of second-order accuracy has been obtained by computing the ratio of the errors (as a function of time) of those with $2 m$ time-steps to those 
with $m$ time-steps for a given final value of $\tau$. In all computations, a moderate degree of discretization in the $\theta$-variable has been found adequate, because of the spectral accuracy in $\theta$-differentiation by FFT. The computations have been performed in the Gowdy lineelement, using the freedom of space-time slicing in the present formulation. Numerical results and convergence data have been obtained for the polarized, pseudo-unpolarized and the two unpolarized Gowdy waves. The results are shown in Figs. 1-3, for both the polarized wave (Figure 1) and two unpolarized waves (Figure 2,3). The results show second-order convergence in accord with the numerical scheme. Also shown is convergence in the errors in the Ricci tensor (also second-order). The results suggest that more advanced time-stepping algorithms should be applicable, to adapt for for more accurate, long-time computations. At this stage, the results do not indicate a need for implicit time-stepping.

The computational problem of binary coalescence of neutron stars or black holes requires a continuing development, including an extension to three-dimensions, adaptation to nonperiodic boundary conditions, and possibly a minimization of the error in the Ricci tensor.

Two directions for further exploration stand out, which are motivated by the structure of the equations. Firstly, the present formulation offers the first strictly-hyperbolic formulation with complete freedom of foliation (in particular, there is no restriction on the equivalent Hamiltonian lapse function). It will be of interest to exploit this for new avenues in the treatment of horizon boundary conditions, and possibly in the extraction of wave forms at the outer boundary as well. Secondly, the implementation is based on the four-divergence of the Riemann tensor and contains no Christoffel symbols. Somewhat analogous equations are given by Faraday's equations, which recently have been successfully implemented using cylindrical coordinates in simulations of magnetized relativistic jets [5, [7]. Here, the notorious axis instabilities are fully regularized [6], whence it will be of interest to extend this regularization to the present equations. Cylindrical coordinates would be desirable in view of the expected simplification in the asymptotic wave form at large distances.

Finally, inclusion of a non-trivial stress-energy tensor is required in the problem of coalescence of neutron stars and possibly black holes as well [8]. 
Acknowledgment. The author greatfully acknowledges stimulating discussions with Saul Teukolsky. This work has been supported in part by NSF grant $94-08378$ and by the Grand Challenge grant NSF PHY 93-18152/ASC 93-18152 (ARPA supplemented). The Cornell Theory Center is supported by NSF, NY State, ARPA, NIH, IBM and others.

Appendix. The analytical solution of the polarized Gowdy wave is given by $P_{0}(\tau, \theta)$ in (8) and $\lambda(\tau, \theta)$ in (10). It has been found useful in debugging the program and in studying convergence to be able to monitor each and every variable during the simulation in comparison with the exact solution. To obtain these and all derived quantities, such as $\omega_{a \mu \nu}$ and $R_{a b \mu \nu}$, at every time-step, numerical evaluation of the right hand-sides of (8) and (10) are needed. A convenient method to do so is by a direct evaluation of their defining expressions in terms of the metric, using numerical differentiation by finite differences across small timeand space-intervals $\epsilon$ (much smaller than the time-step size and spatial discretization in the numerical integration of the wave equations). This requires accurate evaluation of

$$
\begin{aligned}
T(\tau) & =\frac{1}{2} \int_{e^{-\tau}}^{1}\left\{Y_{0}^{\prime 2}(t)+Y_{0}^{2}(t)\right\} t \mathrm{~d} t \\
& =\frac{1}{2} \int_{0}^{\tau}\left\{Y_{0}^{\prime 2}\left(e^{-\tau}\right)+Y_{0}^{2}\left(e^{-\tau}\right)\right\} e^{-2 \tau} \mathrm{d} \tau
\end{aligned}
$$

up to its second derivatives about each of the $\tau_{n}=n \Delta \tau$.

Generally, $T(\tau)$ on $\left[\tau_{a}, \tau_{b}\right]$ is given accurately up to its $k$-th derivatives in each subinterval $D_{n}=\left[\tau_{n}-k \epsilon, \tau_{n}+k \epsilon\right]$ about $\tau_{n}=n \Delta \tau=\frac{\tau_{b}-\tau_{a}}{m}$ by a chain of function elements $\left\{f_{n}, D_{n}\right\}$ is used (see, e.g. [10]). Here, the $f_{n}$ are polynomial approximations

$$
f_{n}(\tau)=c_{n 0}+c_{n 1}\left(\tau-\tau_{n}\right)+c_{n 2}\left(\tau-\tau_{n}\right)^{2}+\cdots
$$

to $T(\tau)$ on $D_{n}$, and the $D_{n}$ cover $[-k \epsilon, m \Delta \tau+k \epsilon]$. Naturally, the degree of the $f_{n}$ is chosen to be greater than or equal to $k$. In the case at hand, $k=2$, and the degree of $f_{n}$ is chosen to be three. 
The coefficients $c_{n l}, l=1,2, \cdots$ follow by direct evaluation of the (analytic) prescription of the integrand of $T(\tau)$ and its (analytical) derivatives. A set of accurate values of the $c_{n 0}$ then follows by first evaluating $\left\{T\left(\tau_{k}^{\prime}\right)\right\}$ on

$$
\tau_{k}^{\prime}=\frac{1}{2} m \Delta \tau^{\prime}\left(1+\cos x_{k}\right), x_{k}=\frac{2 \pi}{N},
$$

using integration of its integrand on the $\left\{\tau_{k}^{\prime}\right\}$ by the Fast Fourier Transform, where $N$ is the degree of the FFT, followed by an interpolation of the $T\left(\tau_{k}^{\prime}\right)$ to the $T\left(\tau_{n}\right)$ using the $c_{n l}$, $l=1,2, \cdots$.

In our computations $N=256$, which gives results on $T(\tau)$ to within machine accuracy. Of course, for every derived quantity involving numerical differentiation, there is a loss of accuracy determined by the numerical differentiation parameter $\epsilon$. We have chosen $\epsilon=10^{-6}$. 


\section{REFERENCES}

[1] C. Bradaschia, E. Caloni, M. Cobal, R. Del Fasbro, A. Di Virgilio, A. Giazotta, E. Holloway, H. Kautzky, B. Michelozzi, V. Montelatici, D. Pascuello, W. Velloso, in Gravitation 1990, Proceedings of the Banff Summer Institute, Banff, Alberta, edited by R. Mann \& P. Wesson (World Scientific, Singapore, 1991).

[2] Jackson. CLassical ELectrodynamics. John Wiley \& Sons, New York, 1975.

[3] B.K. Berger \& V. Moncrief. Phys. Rev. D, 48(10):4676-4687, 1993.

[4] G. Strang. Linear Algebra and its applications. Academic Press, New York, 1980.

[5] M.H.P.M. van Putten. Proc. Cornelius Lanczos Int. Centenary Conf., edited by J.D. Brown, M.T. Chu, D.C. Ellison and R.J. Plemmons, p449 (SIAM, Philadelphi, 1994).

[6] M.H.P.M. van Putten. Int. J. Bifurcation 6 Chaos, 4(1):57-69, 1994.

[7] M.H.P.M. van Putten. 467:L57, 1996.

[8] van Putten M.H.P.M. Phys. Rev. D, 54(10):R5931, 1996.

[9] van Putten M.H.P.M. \& Douglas M. Eardley. Phys. Rev. D, 53(6):3056, 1996.

[10] Ruding W. Real \& Complex Analysis. McGraw-Hill, New York, 1987.

[11] A. Abramovici, W.E. Althouse, R.W.P. Drever, Y.Gursel, S.Kawamura, F.J. Raab, D. Shoemakes, L. Siewers, R.E. Spero, K.S. Thorne, R.E. Vogt, R. Weis, S.E. Whitcomb \& M.E. Zucker. Science, 256:325, 1992. 


\section{FIGURES}

FIG. 1. Shown is the simulation on $0 \leq \tau \leq 5.12$ of the polarized Gowdy wave. Distributions $\lambda(\tau, \theta)$ and $P(\tau, \theta)$ are displayed (upper windows), together with their $\theta$-distribution at $\tau=5.12$ (middle windows). Errors are obtained in computations with a consecutive doubling of the number of time-steps ( $m=512,1024,2048)$, and are given as a function of time in the lower window to the left for both $P$ and $\lambda$ (o for $m=512, \times$ for $m=1024$ and lines for $m=2048$ ). The errors show proper second order convergence (lower window to the right), obtained upon dividing the errors for $m=1024$ by those of $m=512(\times)$, and those for $m=2048$ by those of $m=1024$ (lines). These errors have been computed with reference to the analytical solution to Gowdy's reduced wave equations.

FIG. 2. Shown is the simulation on $0 \leq \tau \leq 5.12$ of the unpolarized Gowdy wave in response to initial data with $A=B=1$. Discretization in $\theta$ is $n=64$ (32 points displayed). Errors are obtained in computations with a consecutive doubling of the number of time-steps $(m=512,1024,2048)$, and are given as a function of time in the lower window to the left for each of the three functions $P, Q$ and $\lambda$ (o for $m=512, \times$ for $m=1024$ and lines for $m=2048$ ). The errors show proper second order convergence (lower window in the middle), obtained upon dividing the errors for $m=1024$ by those of $m=512(\times)$, and those for $m=2048$ by those of $m=1024$ (lines). The errors in the Ricci tensor for the three discretizations (lower window to the right) likewise show second order convergence. An additional dashed line further shows the error in the Ricci tensor for extremely high time-discretization, $m=32768$. These errors are computed using numerical results from Gowdy's reduced wave equations for comparison.

FIG. 3. Shown is the simulation on $0 \leq \tau \leq 7.68$ of the unpolarized Gowdy wave in response to initial data with $A=0$ and $B=1$. Discretization in $\theta$ is $n=64$ (32 points displayed). The windows display the same variables and errors as described in the previous figure, with errors again computed using results from Gowdy's reduced wave equations for comparison. A notable transition occurs in the behavior of the errors, specifically in those of the Ricci tensor, as steep gradients are formed in $P$ and $Q$ (and to a lesser extend in $\lambda$ as well) about $\tau=5$. 

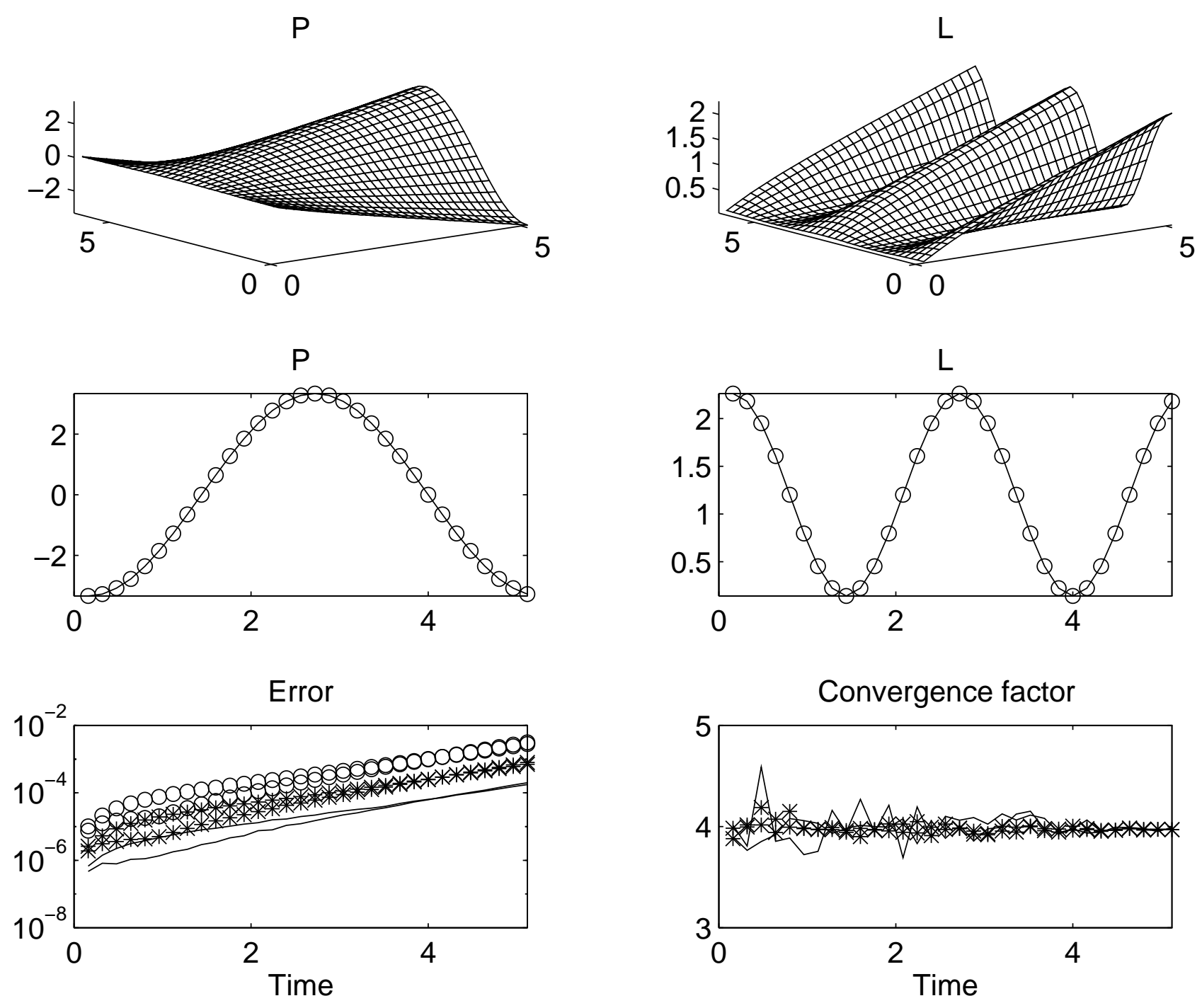

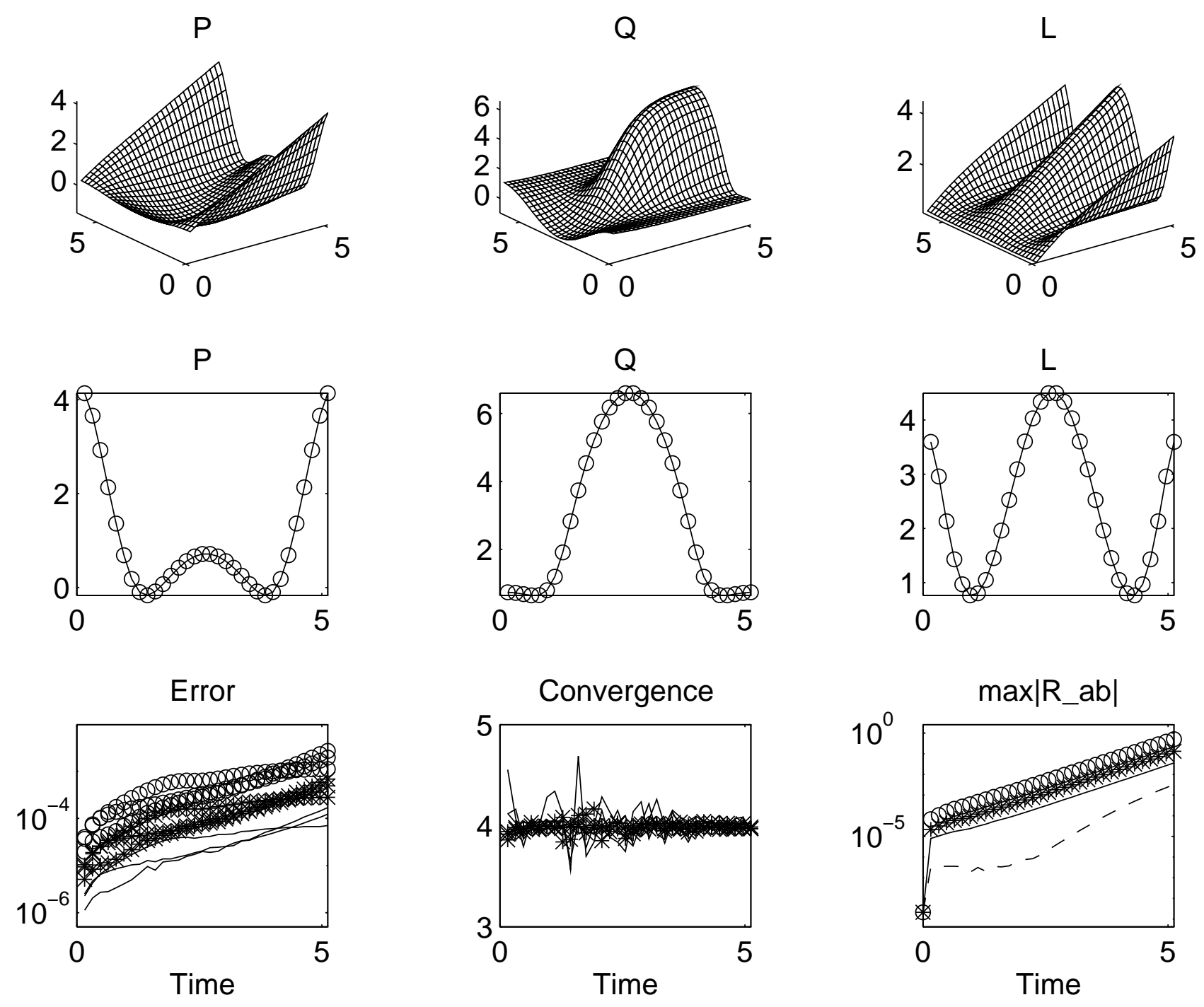

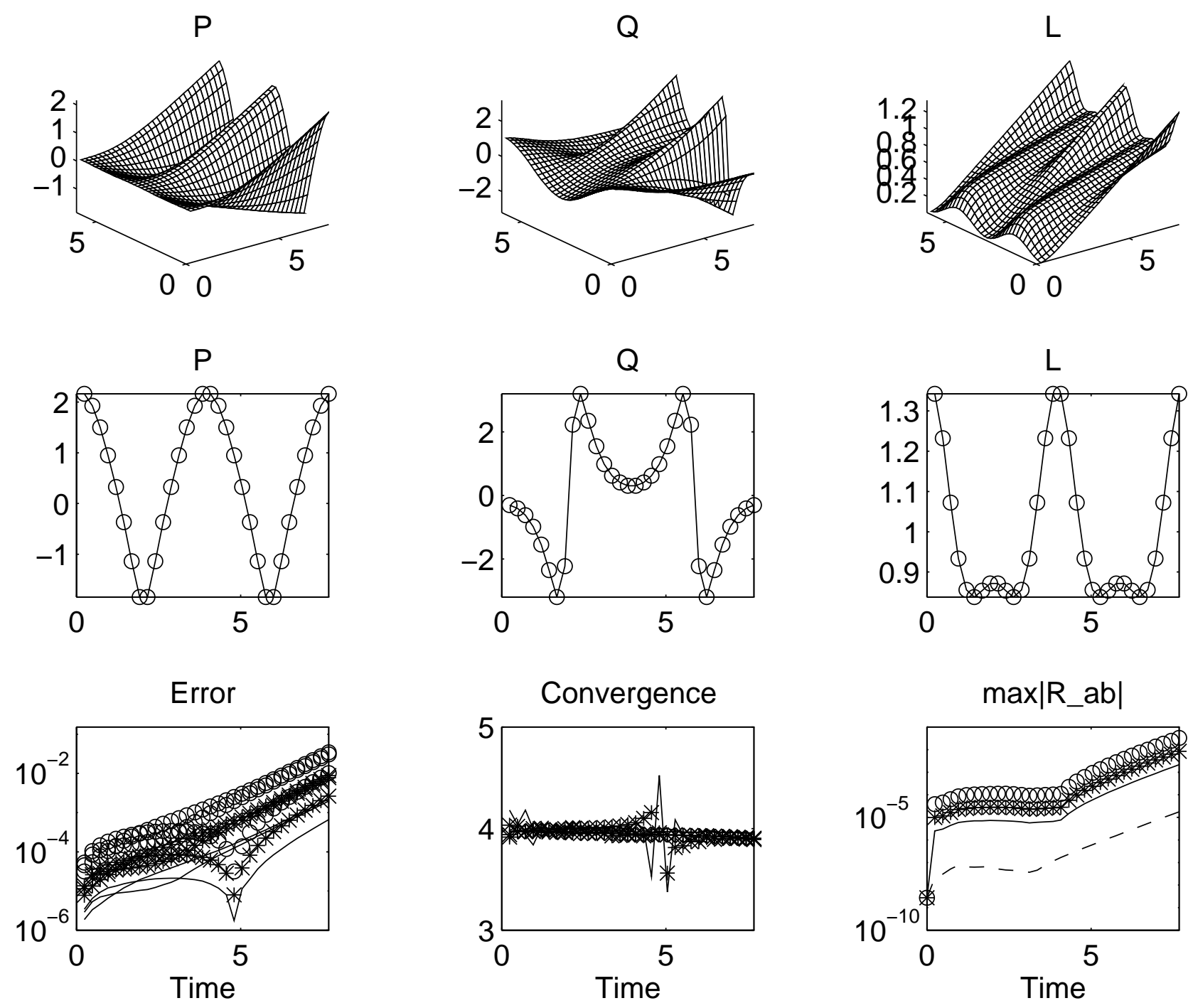